\title{
Noise fiber lasers
}

\author{
Y. O. Barmenkov, P. Muniz-Cánovas, and A. V. Kir’yanov \\ Centro de Investigaciones en Óptica, \\ A.C., Loma del Bosque 115, Col. Lomas del Campestre, León, Guanajuato 37150, México.
}

\author{
J. A. Minguela-Gallardo \\ División de Ciencias e Ingenierías, Universidad de Guanajuato, \\ León, Guanajuato 37150, México.
}

G. Beltrán-Pérez

Facultad de Ciencias Físico-Matemáticas, Benemérita Universidad Autónoma de Puebla, Puebla 72000, México.

J. L. Cruz and M. V. Andres

Departamento de Física Aplicada Instituto de Ciencia de Materiales, Universidad de Valencia, 46100 Valencia, Spain.

Received 11 December 2020; accepted 21 January 2021

In this paper, we present a brief review of the noise operation mode of fiber lasers. These lasers were studied recently by a collaborative group that includes researchers, professors, and Ph.D. students from the Centro de Investigaciones en Óptica, A.C. (Leon, Guanajuato, Mexico) and from the Universidad de Valencia (Valencia, Spain). Meanwhile, the pioneer works in this topic important for understanding the physics behind fiber lasers' operation and for practical applications were done with the active participation of Dr. Evgeny Kuzin from the Instituto Nacional de Astrofísica, Óptica y Electrónica (Puebla, México) and Dr. Georgina Beltrán-Pérez from the Benemérita Universidad Autónoma de Puebla (Puebla, Mexico), whose Ph.D. study was supervised by him. The fiber lasers under study were based on commercial erbium- and ytterbium-doped fibers as gain media and operated in continuous-wave and actively Q-switched regimes. All these fiber lasers were arranged in Fabry-Perot cavity configuration with fiber Bragg gratings as narrow-band reflectors. In the case of actively Q-switched lasers, a standard fiberized acousto-optic modulator was placed close to the rear (100\%) reflector. The most important conclusion of all the works grounding the present review is that, independently of laser operation regime, continuous wave, or actively Q-switched, these fiber lasers operate in the extreme noise regime with the photon statistics described by Bose-Einstein distribution inherent to narrow-band thermal light sources.

Keywords: Fiber lasers; photon statistics; Bose-Einstein distribution.

En este artículo presentamos una breve revisión de la operación en modo de ruido de los láseres de fibra. Dichos láseres han sido estudiados recientemente por un grupo colaborativo que incluye investigadores, profesores y estudiantes de doctorado del Centro de Investigaciones en Óptica A.C. (en León, Guanajuato, México) y de la Universidad de Valencia (en Valencia, España). Además, los trabajos pioneros sobre el tema, importantes para entender la física detrás de la operación de estos láseres y sus aplicaciones prácticas fueron, realizados con la participación del Dr. Evgeny Kuzin del Instituto Nacional de Astrofísica, Óptica y Electrónica (Puebla, México) y la Dra. Georgina BeltránPérez de la Benemérita Universidad Autónoma de Puebla (Puebla, México), cuyo estudio de doctorado fue asesorado por él. Los láseres de fibra sujetos del estudio fueron realizados con base en fibras comerciales dopadas de erbio e iterbio como medios de ganancia y operaron en regímenes tanto de onda continua como de conmutación Q activa con desplazamiento de frecuencia. Todos estos láseres estuvieron arreglados en una configuración de la cavidad Fabry-Perot con rejillas Bragg de fibra como reflectores de banda estrecha. En el caso de los láseres de conmutación Q activa, se utilizó un modulador acusto-óptico de fibra estándar cerrado del lado del reflector posterior (de 100\%). La conclusión más importante de todos los trabajos resumidos es que, independientemente del régimen de operación láser, onda continua o con conmutación Q activa, los láseres operan en el régimen de ruido extremo con estadísticas de fotones descritas por la distribución Bose-Einstein, inherente a las fuentes de luz termal de banda estrecha.

Descriptores: Láseres de fibra óptica, estadística de fotones, distribución Bose-Einstein.

PACS: 42.55.Wd; 42.50.Ar; 42.60.Mi.

\section{Introduction}

Fiber lasers (FLs) are attractive devices that found a lot of commercial applications. Such light sources are usually based on fully fiberized schemes and demonstrate high optical and electrical to optical budgets. FLs operate in a great variety of regimes, characterized by very narrow (singlefrequency FLs) [1] to extremely large (supercontinuum FLs)
[2] optical spectra. FLs can operate in continuous-wave (CW) and pulsed (Q-switched, mode-locked, and soliton) regimes, in which pulse widths vary in the range from hundreds of nanoseconds to tens of femtoseconds [3-7].

Although such regimes of FLs as CW and actively Qswitching (AQS) are deeply explored, and many papers on the matter are published, some particularly important charac- 
teristics of them, including photon noise, are not yet studied in detail due to limitations associated with the lack of highfrequency receiving equipment.

In this paper, we summarize our recent results on studying extremely high photon noise inherent in FLs operated in $\mathrm{CW}$ and AQS regimes. Given that such FLs are implemented in the simplest Fabry-Perot cavity configuration with narrowband reflectors based on fiber Bragg gratings (FBGs), their optical spectra are so narrow that are comparable with or even less than radiofrequency (RF) band of the modern receivers, usually measured by tens GHz. In such circumstances, both types of FLs demonstrate notable photon noise, described by $M$-fold degenerate Bose-Einstein photon distribution, where $M$ is the degeneracy factor dependent on the ratio of the optical band $\left(B_{o p t}\right)$ to the electrical one $\left(B_{e l}\right)$ and the smaller this ratio, the closer $M$-factor to 1 for polarized light. In turn, for randomly polarized light, $M$-factor is limited from below by 2 (per two orthogonal polarizations).

In this review, some aspects of noise FLs are highlighted; as the matter was not once discussed with Dr. Kuzin, we dedicate it to his memory.

\section{Experimental arrangements}

The lasers' schemes are sketched in Fig. 1: the CW and AQS FL designs are shown in the upper (a) and lower (b) panels. In both cases, the laser cavity consists of two home-made FBGs, of $100 \%$ (rear FBG) and $\sim 10 \%$ (output FBG) reflectivity. The amplifying medium in the first scheme is standard double-clad ytterbium-doped fiber (DC-YDF, Nufern, model SM-YDF-5/130-VIII), and that in the second scheme is single-clad erbium-doped fiber (EDF, Liekki, model ER16$8 / 125)$.

The YDF laser (YDFL) was pumped by laser diodes operating at $975 \mathrm{~nm}$ through a multimode pump combiner, whereas the EDF laser (EDFL) was pumped by a laser diode at $1480 \mathrm{~nm}$ through a single-mode wavelength-division multiplexer (WDM). Small-signal clad absorption of YDF at pump wavelength is $1.66 \mathrm{~dB} / \mathrm{m}$, and small-signal in-core absorption of EDF at pump wavelength is $\sim 7 \mathrm{~dB} / \mathrm{m}$. The


FIGURE 1. Experimental arrangements of a) CW ytterbium-doped fiber laser and b) actively Q-switched erbium-doped fiber laser. active fiber lengths were chosen to be (a) $15 \mathrm{~m}$ (YDF) for which pump power is absorbed by $\sim 25 \mathrm{~dB}$ in double-clad pump configuration used and (b) $4 \mathrm{~m}$ (EDF) for which parasitic lasing at the laser wavelength is yet incapable to develop, given tiny reflection $(-50 \mathrm{~dB})$ from a blocked acousto-optic modulator (AOM). AOM was controlled using a fast pulse generator (A-A Opto-Electronic, model MT110-IIR25-3FioSM5-J1-A) with a rise-time of $25 \mathrm{~ns}$ and 110-MHz one-pass frequency shift.

The YDFL and EDFL operated at $1061 \mathrm{~nm}$ and 1550 nm, respectively. The YDFL efficiency was nearly $70 \%$, and the peak pulse power of the actively Q-switched EDFL was 500 W. A fiber polarizer was placed on the lasers' outputs for studying statistical properties of the transmitted polarized light.

\section{Theoretical background for studying the laser photon statistics}

It will be shown in the following sections that the FLs under study are characterized by the photon statistics described by the $M$-fold Bose-Einstein distribution. $M$-factor is the degeneracy factor that is equal to the number of independent states of amplified spontaneous emission (ASE) within the laser optical spectrum [8-10]:

$$
M=s \frac{\pi \xi^{2}}{\pi \xi \operatorname{erf}(\sqrt{\pi} \xi)-\left[1-\exp \left(-\pi \xi^{2}\right)\right]}
$$

where $s$ is the number of orthogonal linear polarization states ( 1 or 2 ) at the photodetector input, $\xi=B_{o p t} / B_{e l}$ is the ratio of the laser optical spectrum width (in $\mathrm{Hz}$ ) to the RF band of the photo-receiving equipment, and erf is the Gauss error function. In turn, $M$-fold Bose-Einstein distribution is described as follows [8-10]:

$$
P(n, \bar{n}, M)=\frac{(n+M-1) !(\bar{n})^{n}}{n !(M-1) !(1+\bar{n})^{n+M}}
$$

where $P(n, \bar{n}, M)$ is the probability of counting $n$ ASE photons by photodetector during the integrating time $T=1 / B_{e l}$ and $(\bar{n})$ is the mean photon count ( $n$ and $\bar{n}$ are proportional, accordingly, to the instant laser power, $P(t)$, and the mean laser power, $\left.P_{\text {mean }}\right) ; B_{e l}$ is the cumulative RF band of the set comprising the photodetector and the oscilloscope, used in the experiments.

Figure 2a) shows the dependence of $M$-number versus for two $s$-values: $s=1$ (linearly polarized light, red curve) and $s=2$ (randomly polarized light, blue curve). It is seen that when the laser optical bandwidth is much less than the photodetector's RF bandwidth, $M$-value tends to approach 1 and 2 at $s=1$ and $s=2$, respectively. Note that the extreme cases of $M=1$ (polarized light) and $M=2$ (unpolarized light) cannot be physically realized as they correspond to the null optical width of a laser spectrum $\left(B_{o p t}=0\right)$. When the laser optical spectrum is broad $(\xi \gg 1), M$-value is equal to $s$. The dash lines in this figure present the function $s$ for comparison. 

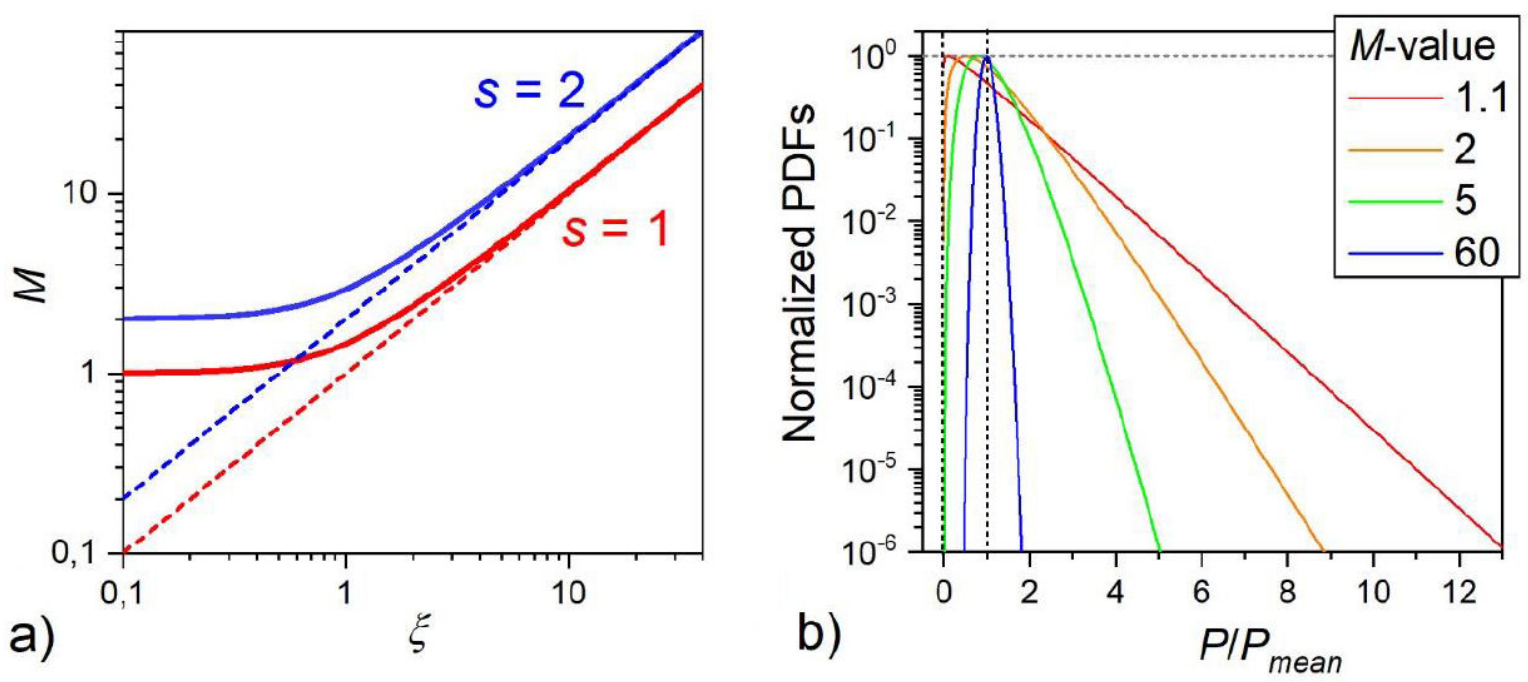

FIGURE 2. a) $M$-value $v s . \xi$. b) Normalized probability density function (PDF) of photon count $v s$. normalized instant laser power.
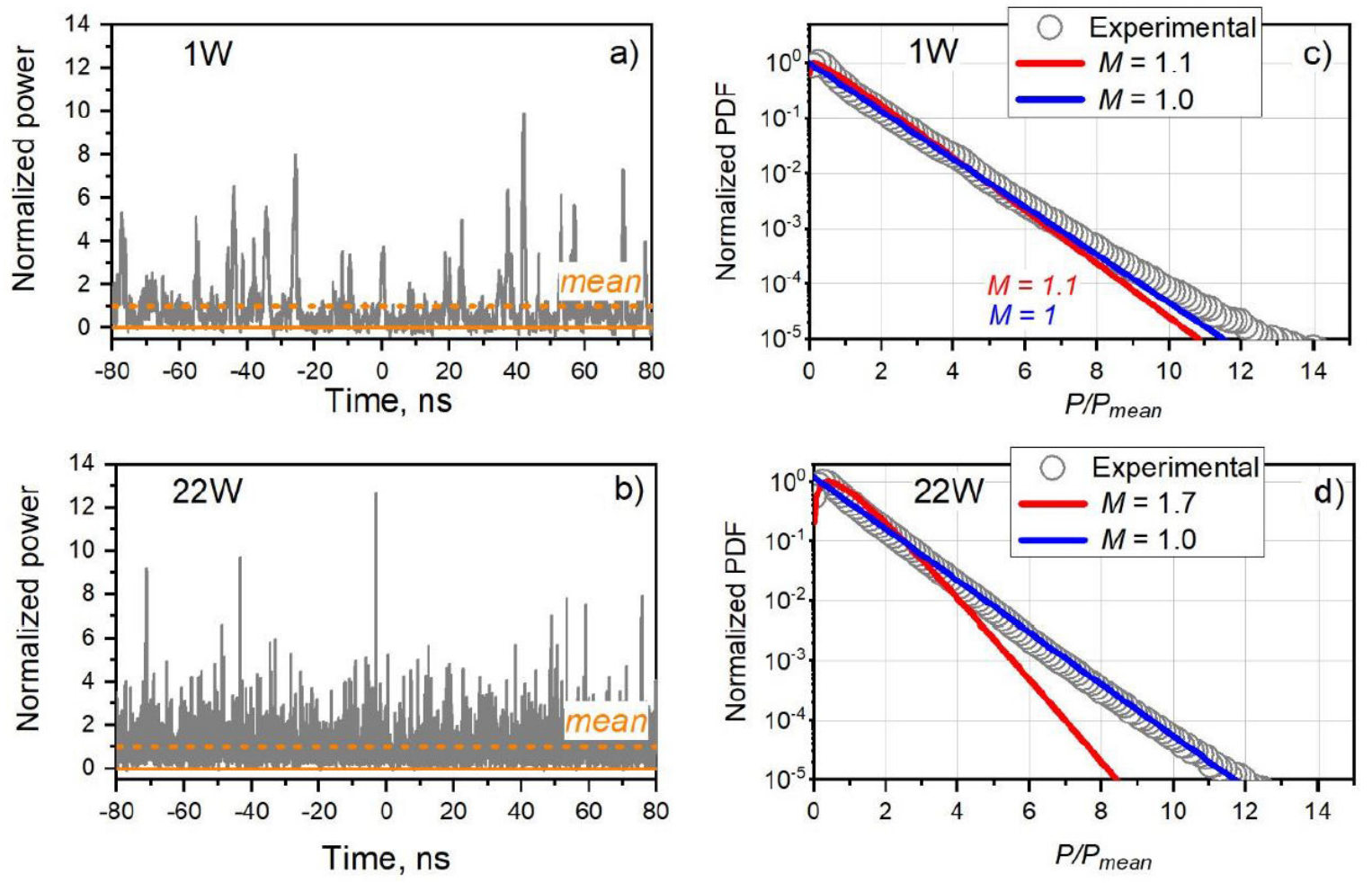

FIGURE 3. YDFL noise trains measured at a laser power of a) $1 \mathrm{~W}$ and b) $22 \mathrm{~W}$ for polarized light. The mean laser power is shown by the dash orange line and the zero-signal by the solid orange line. Normalized PDFs of photon counts of polarized laser signals measured at c) $1 \mathrm{~W}$ and d) $22 \mathrm{~W}$ of laser power.

Figure $2 \mathrm{~b}$ ) shows the normalized probability density function (PDF) of photon count as a function of the normalized instant laser power for several $M$-values (indicated in the inset). It is seen that at a small $M$-value ( $M=1.1$ ), the PDF function is virtually exponential (or linear in the semi-log plot). When the laser optical bandwidth is comparable with or larger than the photodetector RF band, the PDF function demonstrates a peak that moves to the position
$P / P_{\text {mean }}=1$ at its increasing to the large values $(M \gg 1)$. The position of the PDF peak that corresponds to the maximum photon count is observed at the following normalized laser power [11]:

$$
\frac{P}{P_{\text {mean }}}=\frac{(M-1)}{M}
$$


When $M$-value is varied between 1.1 and a few tens, the PDF function is strongly asymmetrical. When $M$ is further increased up to 50 and higher, the PDF function gets more symmetrical, and at the limit $M \rightarrow \infty$, it obeys the Poisson distribution that described the photon statistics for the optical band strongly exceeding the photodetector's RF band.

\section{Photon noise in CW YDFL}

Our experiments with the CW YDFL have shown an excessive photon noise in the laser signal in the whole range of output powers (from $1 \mathrm{~W}$ to $22 \mathrm{~W}$ ); see Fig. 3, where the laser noise trains are shown for $1 \mathrm{~W}$ and $22 \mathrm{~W}$ outputs (polarized light). In this experiment, we used a $25-\mathrm{GHz}$ photodetector and a 16-GHz real-time oscilloscope. No classic $\mathrm{CW}$ regime, characterized by longitudinal mode beating / competition and relaxation oscillations [12], was observed in this case. From these trains of the noise signal, one can see that some noise peaks arising with low probability highly exceed (by more than an order of magnitude) the mean laser power. Moreover, at increasing laser power, the noise peaks happen more frequently, and their effective width dramatically decreases (see Ref. [10]). In the meantime, the YDFL spectra broaden with increasing the laser power (see Fig. 4), an effect associated with the fiber optical nonlinearity at relatively high laser power [13].

The narrowest laser spectrum was observed at the lowest laser power $(1 \mathrm{~W})$, at which the spectrum width is eventually defined by that of the output narrow-band low-reflection FBG (FWHM $30 \mathrm{pm}$ ). Note that the spectrum width was obtained as deconvolution of the FBG spectrum, measured with the OSA, and the OSA's spectral response to a single-frequency laser at $\sim 1060 \mathrm{~nm}$. At the highest laser power $(22 \mathrm{~W})$, the laser spectrum broadens up to $80 \mathrm{pm}$ [10]. From here, one can conclude that the $M$-factor grows from $\sim 1.1$ to $\sim 1.7$ with increasing laser power from $1 \mathrm{~W}$ to $22 \mathrm{~W}$.

Generally, the YDFL photon noise can be described by the $M$-fold Bose-Einstein distribution. Given that $M$-factor at the lowest laser power is approximately equal to the unit $(M=1.1)$ while it noticeably increases at the highest power

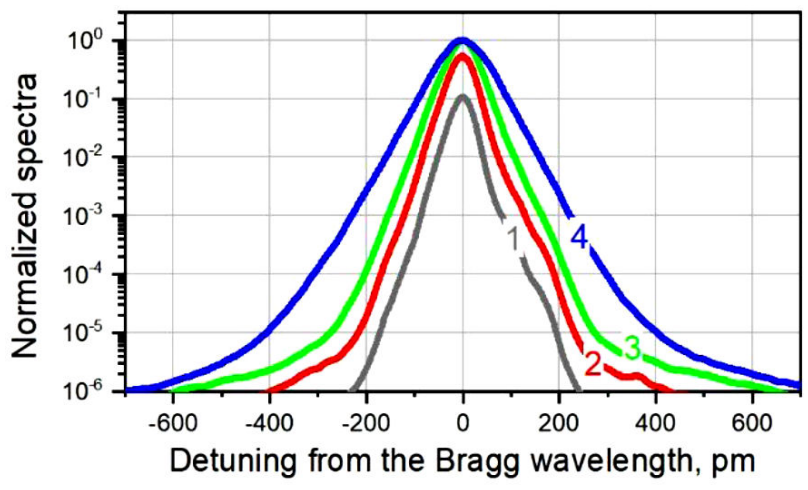

FIGURE 4. YDFL spectra at different laser powers: (1) $1 \mathrm{~W},(2)$ $6 \mathrm{~W}$, (3) $14 \mathrm{~W}$, and (4) $22 \mathrm{~W}$. The spectra are shown as a detuning from the laser peak wavelength $(1061 \mathrm{~nm})$ and are normalized to the spectral maximum measured at laser power of $22 \mathrm{~W}$. (up to $M=1.7$ ), the PDF of photon count ought to be almost exponential while strongly asymmetrical in these two cases (see Fig. 2b)). However, as seen from Figs. 3c), d), in both the histogram of photon count is well approximated by the nearly exponential PDF, corresponding to a single independent ASE mode. For comparison, in each plot, we show two curves, simulated for the $M$-values found from the laser optical spectra and for the ideal case, $M=1$ (see the insets for $M$-values used). The existence of the solitary ASE mode for polarized light can be explained by the following circumstance: the output FBG with narrow optical spectrum reflects the narrow-band laser light with photon statistics with $M \approx 1$, and the nonlinear fiber length is much shorter than the fiber dispersion length [10]. Thus, even though the nonlinear interaction of the laser light with the optical fiber is strong, this does not lead to any pronounced change in the photon statistics.

\section{Photon noise in AQS EDFL}

The AQS EDFL (refer to Fig. 1b)) demonstrates the noise behavior like the CW YDFL, discussed above. Figure 5 shows the single-shot trace of the noise Q-switched, pulse and, for comparison, the pulse averaged over 512 scans. Note that in this type of lasers Q-switched pulse consists of a train of subpulses, with the number of developed sub-pulses and their powers depending on the EDF initial charge (viz. the multiplication of the density of excited $\mathrm{Er}^{3+}$ ions in the core with the laser level energy) and cavity loss [14-16]. Here, the width of the output $1 \mathrm{~cm}$ FBG was $80 \mathrm{pm}$.

The sub-pulses are separated by the round-trip time of the laser wave traveling along the cavity ( $~ 60 \mathrm{~ns})$; the most powerful sub-peak is the fifth one (the main sub-pulse), measured from the moment of opening the AOM. One can see from this figure that some of the noise spikes within the highest sub-pulse are by an order of magnitude greater than the mean peak power. Note that we did not use a polarizer at the detector input in this experiment, so the laser signal had random polarization $(s=2)$.



FIGURE 5. The laser pulses: single-shot train is shown by the grey curve and the averaged signal by the red curve. 


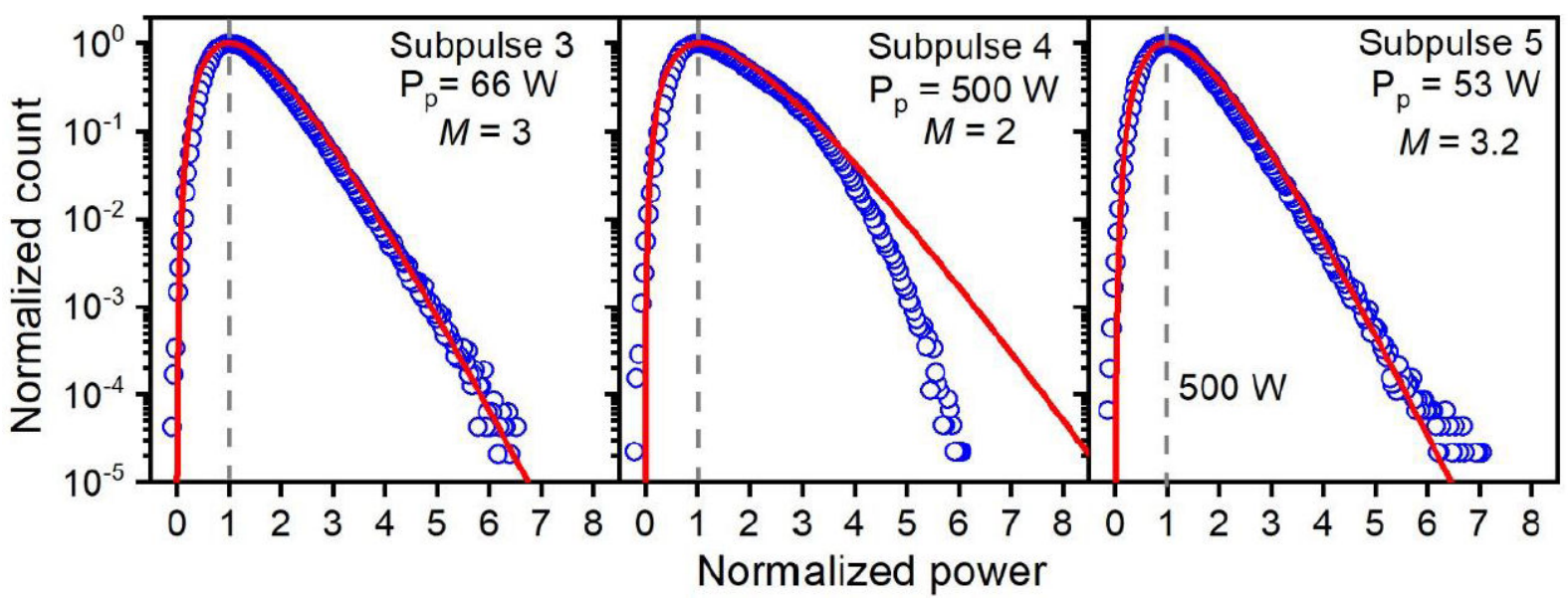

FIGURE 6. Experimental PDFs of photon count for the three most powerful sub-pulses (indicated in each plot). Laser power is normalized to the most probable power that corresponds to the maximal values of the histograms. $P_{p}$ means peak pulse power.

Each sub-pulse of which Q-switched pulse consists can be characterized by its photon statistics. Figure 6 demonstrates such statistics for the three most powerful sub-pulses $(3,4$, and 5). The powers of the pulses vary from tens of watts to $0.5 \mathrm{~kW}$ for the main sub-pulse.

As seen from this figure, the experimental PDFs can be fit with a high precision by the Bose-Einstein distribution with $M$-factors indicated in each plot. From these, one can reveal that the smallest $M$-number $(M=2)$ is attained for the most powerful pulse, whereas for the less powerful ones, $M$-number increases. Note that since the experiments with the EDFL were carried out with randomly polarized light $(s=2)$, the value $M=2$ characterizes a single independent ASE state for each orthogonal linear polarization (compare with the YDFL, discussed above). Also, note that when normalized power is higher than 3 (the laser instant power is higher than $\sim 1.5 \mathrm{~kW}$ ), the histogram is cut due to the nonlinear effects resulted in ongoing supercontinuum (SC) generation. It deserves mentioning that AQS EDFLs are nowadays successfully applied to generate SC [16,17].

\section{Conclusions}

In this paper, we reported the photon noise statistics of $\mathrm{CW}$ and actively Q-switched ytterbium- and erbium-doped fiber lasers. It was shown that in the case of $\mathrm{CW}$ ytterbiumdoped fiber laser, no classical $\mathrm{CW}$ regime where longitudinal modes compete, and relaxation oscillations develop is observed. Furthermore, both fiber lasers operate as multi-pass amplifiers of narrow-band spontaneous emission with excessive photon noise, described by the $M$-fold Bose-Einstein distribution that is inherent to narrow-band thermal light. Interestingly, some spikes of the photon noise are in magnitude by an order greater than the mean laser power in CW ytterbium fiber laser and the average pulse power in actively Q-switched erbium fiber laser. The reported results may be important for a deeper understanding of the physics of fiber lasers.
1. S. Fu et al. Review of recent progress on single-frequency fiber lasers [Invited]. J. Opt. Soc. Am. B 34 (2017) 1407. https: //doi.org/10.1364/JOSAB.34.000A49

2. R. R. Alfano, The supercontinuum laser source: the ultimate white light. 3rd edition (New York: Springer, 2016).

3. D. J. Richardson, J. Nilsson, and W. A. Clarkson, High power fiber lasers: Current status and future perspectives [Invited]. J. Opt. Soc. Amer. B, Opt. Phys. 27 (2010) B63. https: //doi.org/10.1364/JOSAB.27.000B63

4. Y. O. Barmenkov, A. V. Kir'yanov, J. L. Cruz, and M. V. Andrés, Pulsed Regimes of Erbium-Doped Fiber Laser QSwitched Using Acousto-Optical Modulator. IEEE J. Sel. Topics Quant. Electron. 20 (2014) 0902208. DOI: 10.1109/ JSTQE.2014.2304423
5. C. Cuadrado-Laborde et al. Transform-limited pulses generated by an actively Q-switched distributed fiber laser. Opt. Lett. 33 (2008) 2590. https://doi.org/10.1364/OL.33. 002590

6. P. K. Cheng et al. Ultrafast soliton and stretched-pulse switchable mode-locked fiber laser with hybrid structure of multimode fiber based saturable absorber. Scientific Reports 9 (2019) 10106. DOI: $10.1038 / \mathrm{s} 41598-018-34762-4$

7. L. Wang et al. Femtosecond mode-locked fiber laser at 1 $\mu \mathrm{m}$ via optical microfiber dispersion management. Scientific Reports 8 (2018) 4732. https://doi.org/10.1038/ s41598-018-23027-9

8. J. W. Goodman, Statistical Optics (New York: Wiley, ch. 6, 2000). 
9. S. M. Pietralunga, P. Martelli, and M. Martinelli, Photon statistics of amplified spontaneous emission in a dense wavelength-division multiplexing regime. Opt. Lett. 28 (2003) 152.https://doi.org/10.1364/OL.28.000152

10. P. Muniz-Cánovas, Y. O. Barmenkov. A. V. Kir'yanov, J. L. Cruz, and M. V. Andrés, Ytterbium-doped fiber laser as pulsed source of narrowband amplified spontaneous emission, Scientific Reports 9 (2019) 13073. https://doi.org/10. $1038 / s 41598-019-49695-9$

11. Y. Barmenkov, A. V. Kir'yanov, P. Muniz-Cánovas, J. L. Cruz, and Miguel V. Andrés, Photon noise in continuous-wave ytterbium-doped fiber laser, Chapter of book Optical fiber technology and applications: resent advantages, IOP Publishing (Bristol, England), to be published.

12. Anthony E. Siegman, Lasers (Sausalito, CA: University Science Books, 1986).
13. Govind P. Agrawal, Nonlinear Fiber Optics. 3rd edition (San Diego: Academic Press, 2001).

14. S. A. Kolpakov et al., Distributed model for actively Qswitched erbium-doped fiber lasers, IEEE J. Quant. Electron. 47 (2011) 928. DOI: $10.1109 /$ JQE . 2011.2143695

15. J. A. Minguela-Gallardo et al., Spectral Dynamics of Actively Q-Switched Erbium-Doped Fiber Lasers, IEEE Phot. Technol. Lett. 29 (2017) 683.

16. J. A. Minguela-Gallardo, Y. O. Barmenkov, A. V. Kir'yanov, and G. Beltrán-Pérez, Photon statistics of actively Q-switched erbium-doped fiber laser, J. Opt. Soc. Amer. B, Opt. Phys. 34 (2017) 1407.

17. P. H. Reddy et al., Fabrication of ultra-high numerical aperture $\mathrm{GeO} 2$-doped fiber and its use for broadband supercontinuum generation, Appl. Opt. 56 (2017) 9315. https: / / doi.org/ $10.1364 / \mathrm{AO} .56 .009315$ 